\title{
IncRNA KCNQ1OT1 may function as a competitive endogenous RNA in atrial fibrillation by sponging miR-223-3p
}

\author{
WEIRAN DAI $^{1^{*}}$, XIAOYING CHAO $^{2 *}$, ZHIYUAN JIANG $^{3}$ and GUOQIANG ZHONG ${ }^{2}$ \\ ${ }^{1}$ Department of Cardiology, The Second Affiliated Hospital of Chongqing Medical University, \\ Chongqing 400010; Departments of ${ }^{2}$ Cardiology and ${ }^{3}$ Hypertension, The First Affiliated \\ Hospital of Guangxi Medical University, Nanning, Guangxi 530021, P.R. China
}

Received July 2, 2021; Accepted October 1, 2021

DOI: $10.3892 / \mathrm{mmr} .2021 .12510$

\begin{abstract}
Atrial fibrillation (AF) is one of the most common forms of cardiac arrhythmia. Novel evidence has indicated that a competing endogenous RNA (ceRNA) mechanism may occur in AF. The present study aimed to identify differentially expressed microRNAs (miRNAs/miRs) in AF and predict their targeting long non-coding RNAs (lncRNAs) to identify a potential ceRNA network involved in AF using bioinformatics analysis. The GSE68475 microarray dataset was downloaded from the Gene Expression Omnibus database and differentially expressed miRNAs in AF were obtained. In addition, right atrial appendage (RAA) tissues from patients with AF were collected to determine the expression levels of the miRNAs identified following bioinformatics analysis using reverse transcription-quantitative PCR ( $n=8$ per group). Subsequently, Gene Ontology (GO) functional term and Kyoto Encyclopedia of Genes and Genomes (KEGG) signaling pathway enrichment analyses of the target genes of differentially expressed miRNAs of interest were performed. The potential upstream lncRNAs targeting the identified miRNAs were predicted using bioinformatics analysis. A dual luciferase reporter assay was used to verify the existence of a targeted relationship between the differentially expressed miRNA and lncRNA of interest. The results identified 43 differentially
\end{abstract}

Correspondence to: Professor Guoqiang Zhong, Department of Cardiology, The First Affiliated Hospital of Guangxi Medical University, 6 Shuangyou Road, Nanning, Guangxi 530021, P.R. China

E-mail: drguoqiang@sina.com

Professor Zhiyuan Jiang, Department of Hypertension, The First Affiliated Hospital of Guangxi Medical University, 6 Shuangyou Road, Nanning, Guangxi 530021, P.R. China

E-mail: drjzy123@sina.com

*Contributed equally

Key words: atrial fibrillation, long non-coding RNA KCNQ1 opposite strand/antisense transcript 1, microRNA-223-3p, competing endogenous RNA expressed miRNAs, including 23 upregulated miRNAs. The trends in the expression levels of miR-223-3p were inconsistent between the microarray data and those recorded in the RAA tissues from patients with persistent AF. Therefore, miR-223-3p was selected as the miRNA of interest for further investigations. The target gene of miR-233-3p was found to be enriched in 57 GO terms and 21 KEGG signaling pathways. According to the bioinformatics prediction, 69 lncRNAs targeting miR-223-3p were identified, including the lncRNA growth arrest-specific transcript 5, lncRNA KCNQ1 opposite strand/antisense transcript 1 (KCNQ1OT1) and lncRNA MYC-induced long non-coding RNA. The results from dual luciferase assay confirmed that miR-223-3p was a direct target of KCNQ1OT1. A ceRNA regulatory relationship may exist between KCNQ1OT1 and miR-223-3p in AF, providing therefore a novel potential research target for further studies.

\section{Introduction}

Atrial fibrillation (AF) is one of the most common forms of cardiac arrhythmia and is accompanied by a high risk of stroke, heart failure and mortality (1). AF is also regarded as a significant contributor towards morbidity and increased healthcare costs (2). The mechanisms underlying AF can be typically classified into three types: Autonomic neural remodeling, electrical remodeling and structural remodeling (3), which can act as triggers or favor the creation of an AF-prone substrate. However, the mechanisms of AF are intricate, which poses challenges for the effective medical intervention of AF.

Recently, multiple molecular factors have been reported to be involved in the pathophysiological progression of AF, including fibrosis, abnormal $\mathrm{Ca}^{2+}$ handling and inflammation (4-6). Previous studies have demonstrated the significant role of microRNAs (miRNAs/miRs) in mediating these molecular factors in AF (7). miRNAs are a type of small non-coding RNA of 19-25 nucleotides in length, and which regulate the expression of target genes at the post-transcriptional level by promoting the degradation of target mRNA or repressing target mRNA translation (8). Zhao et al (9) reported that miR-29a-3p serves roles in the development of AF by downregulating the L-type $\mathrm{Ca}^{2+}$ current (9). Furthermore, Cañón et al (10) demonstrated that the aberrant expression of miR-208b could reduce the expression and function of L-type $\mathrm{Ca}^{2+}$ channel subunits, 
as well as the sarcoplasmic reticulum $\mathrm{Ca}^{2+}$ pump, ATPase sarcoplasmic/endoplasmic reticulum $\mathrm{Ca}^{2+}$ transporting 2, in myocytes isolated from patients with chronic AF, suggesting that miR-208b may be an important mediator in impaired $\mathrm{Ca}^{2+}$ handling during atrial remodeling. Our previous study demonstrated that miR-27b-3p could regulate the Wnt/ $\beta$-catenin signaling pathway and attenuate atrial fibrosis in rats with AF by targeting Wnt3a (11). miRNAs have therefore been considered as promising targets for AF intervention.

Accumulating evidence has indicated that long non-coding RNAs (lncRNAs), a class of non-coding RNAs of $>200$ nucleotides in length, may also serve an important role in cardiac diseases by acting as competing endogenous RNAs (ceRNAs) (12). Salmena et al (13) first proposed the ceRNA theory in 2011, and hypothesized that mRNAs and lncRNAs could communicate with each other by binding to shared miRNAs using miRNA response elements (14). For example, the lncRNA-long intergenic non-protein coding RNA 472 was discovered to promote AF by downregulating the expression of ryanodine receptor 2 via miR-24 (15). However, the underlying regulatory ceRNA mechanism in AF remains to be fully elucidated.

The present study firstly aimed to identify significantly differentially expressed miRNAs in AF by screening microarray data from the Gene Expression Omnibus (GEO) database. Then, right atrial appendage (RAA) tissues from patients with AF were collected to verify the expression of the identified miRNAs. Combined with the validation results, Gene Ontology (GO) functional term and Kyoto Encyclopedia of Genes and Genomes (KEGG) signaling pathway enrichment analyses were performed for the target genes of the differentially expressed miRNAs of interest to fully understand the role of these miRNAs. The potential upstream lncRNAs targeting the identified miRNAs were predicted using Bioinformatics method. Finally, a dual luciferase assay was used to verify whether a targeted relationship existed between significantly differentially expressed miRNAs and lncRNAs of interest. These findings may provide a solid theoretical basis for future research to help identifying potential targets for the treatment of AF.

\section{Materials and methods}

Microarray data collection. The present study used the GEO database (https://www.ncbi.nlm.nih.gov/geo), which is a public gene expression database from the National Center for Biotechnology Information. For microarray retrieval, 'atrial fibrillation' was used as the key word to search for microarrays that had studied the expression levels of miRNAs in AF. The inclusion criteria were as follows: i) Human atrial appendage tissue as the research sample type; ii) the Sinus rhythm (SR) group was set as the control group and the persistent AF group was set as the experimental group, and each group contained $>3$ research samples; and iii) miRNA expression in atrial appendage tissues was the main research focus. The exclusion criteria were as follows: i) Incomplete microarray matrix data; ii) the source of the research sample was unclear; iii) the main research focus of the microarray matrix was not miRNAs; iv) the microarray annotation file was missing or unknown; and v) the microarray data were unable to meet the subsequent data analysis standards after standardized processing. After retrieval, the platform files and microarray data that met the criteria were downloaded for subsequent analysis.

Data preprocessing. To reduce the errors in data analysis, data preprocessing of the downloaded microarray data was performed. Firstly, the corresponding microarray platform files were used to annotate the matrix data of the microarray and convert the miRNA IDs. After annotation, the annotated matrix data were supplemented using the impute toolkit of $\mathrm{R}$ software (version 3.5.1; RStudio, Inc.). All matrix data that did not match the miRNAs were deleted. For the matrix data with the same miRNA ID, the average value was obtained after merging. Finally, Bioconductor's limma toolkit (version 3.10.3; http://www.bioconductor.org/packages/2.9/bioc/html/limma. html) in $\mathrm{R}$ software was used for background correction, provision of missing values and data standardization.

Identification of differentially expressed miRNAs. After data preprocessing, the limma toolkit in $\mathrm{R}$ software was used to identify the significantly differentially expressed miRNAs between AF and SR samples. The screening threshold of significantly differentially expressed miRNAs was set as $\mid \log _{2}$ fold change $(\mathrm{FC}) \mid \geq 0.5$ and $\mathrm{P}<0.05$. The heatmap and volcano map of significantly differentially expressed miRNAs were drawn using $\mathrm{R}$ software. The top four most significantly upregulated and downregulated miRNAs, according to the $\mid \log _{2} \mathrm{FCl}$ values, were selected for verification using reverse transcription-quantitative PCR (RT-qPCR).

Patient studies. We continuously included patients with SR and persistent AF who were hospitalized at the First Affiliated Hospital of Guangxi Medical University (Nanning, China) and were undergoing open-heart surgery for coronary bypass grafting or valve replacement. The patient's electrocardiogram was independently judged by two cardiovascular physicians and combined with medical history. The diagnostic criteria of AF was in line with the diagnostic criteria of AF recommended in the 2016 European Society of Cardiology AF management guidelines (16). Patients were excluded if they had the additional following conditions: left ventricular ejection fraction $<50 \%$, infectious diseases, active myocarditis, endocarditis, active rheumatism, pulmonary disease, hyperthyroidism, hematological diseases, tumors and autoimmune diseases. Since September 2018, RAA tissues were collected at the beginning of surgical intervention under extracorporeal circulation and rapidly cryopreserved in liquid nitrogen, then stored at $-80^{\circ} \mathrm{C}$ until the RAA tissue of the last patient was collected in June 2019. All samples would then be used for RT-qPCR analysis. The experimental protocol was approved by the Ethics Committee of The First Affiliated Hospital of Guangxi Medical University (Nanning, China). Each patient provided written informed consent prior to surgery for RAA tissue collection.

$R T-q P C R$. Total RNA was extracted from the RAA tissues from patients with AF or SR ( $n=8$ per group) using TRIzol ${ }^{\circledR}$ reagent (cat. no. 9108; Takara Bio, Inc.). A NanoDrop ND 1000 spectrophotometer was used to assess the absorbance of the extracted total RNA at wavelengths of 260 and $280 \mathrm{~nm}$. 
The 260/280 ratio was calculated to confirm the quality of the RNA, and only samples with a 260/280 ratio between 1.8 and 2.1 were used. Total RNA was reverse transcribed into cDNA using a tailing reaction kit (cat. no. B532451; Sangon Biotech Co., Ltd.) for miRNA or a reverse transcription kit (cat. no. RR047A; Takara Bio, Inc.) for mRNA according to the manufacturers' instructions. When the universal reverse primers provided by the tailing reaction kit could not complete the cDNA synthesis of miRNA experiment, we replaced the universal reverse primers or redesigned the reverse primers to complete this part of the study. qPCR was subsequently performed on a StepOne Real-Time PCR system (Thermo Fisher Scientific, Inc.). The PCR procedure was as follows: One cycle at $95^{\circ} \mathrm{C}$ for $30 \mathrm{sec}$ to complete the initial denaturation, followed by 40 cycles of $95^{\circ} \mathrm{C}$ for $5 \mathrm{sec}$ and $60^{\circ} \mathrm{C}$ for $30 \mathrm{sec}$ for denaturation, annealing and extension. Melting curve analysis was performed at $\sim 65-95^{\circ} \mathrm{C}$. Expression levels were quantified using the $2^{-\Delta \Delta \mathrm{Cq}}$ method (17) and normalized to GAPDH (for mRNA) or U6 (for miRNA). The sequences of the primers used are listed in Table I.

Bioinformatics analysis. By combining the data from the microarray analysis and patient tissues, the differentially expressed miRNAs of interest were selected for bioinformatics analysis to further explore their functions. GO functional term and KEGG signaling pathway enrichment analyses were conducted for the target genes of the differentially expressed miRNAs of interest $(18,19)$. The GO functional term enrichment analysis consisted of biological process (BP), cellular component (CC) and molecular function (MF) aspects. $\mathrm{P}<0.05$ and a count value $\geq 2$ were used as the significant cutoff values. The results from the GO functional term and KEGG signaling pathway enrichment analyses were sorted using the Revigo toolkit in $\mathrm{R}$ software and visualized in the form of bubble charts.

Prediction of IncRNAs targeting the differentially expressed miRNAs of interest. The miRbase (http://www.mirbase.org) and StarBase (http://starbase.sysu.edu.cn/index.php) databases were used to predict the upstream lncRNAs targeting the differentially expressed miRNAs of interest. The identified lncRNAs were inputted into Cytoscape software (version 3.7.2) to construct the ceRNA network.

Dual luciferase reporter assay. The recombinant luciferase construct, pcDNA3.1-KCNQ1OT1 3'-untranslated region (UTR)-wild-type (WT; Hanbio Biotechnology Co., Ltd.) harboring the WT binding site for miR-223-3p and another recombinantconstruct,pcDNA3.1-KCNQ1OT13'-UTR-mutant (MUT), containing the mutated binding site for miR-223-3p, were constructed. Then, 293T cells (The Cell Bank of Type Culture Collection of The Chinese Academy of Sciences) were co-transfected with the pcDNA3.1-KCNQ1OT1 3'-UTR-WT (KCNQ1OT1 WT) or pcDNA3.1-KCNQ1OT1 3'-UTR-MUT (KCNQ1OT1 MUT) recombinant plasmid and miR-223-3p mimic (sense 5'-UGUCAGUUUGUCAAAUACCCC-3', antisense 5'-GGGGUAUUUGACAAACUGACA-3') or miR-223-3p mimic-negative control (sense 5'-UUUGUACUA CACAAAAGUACUG-3', antisense 5'-CAGUACUUUUGU GUAGUACAAA-3') by Liposomal Transfection Reagent
Table I. Sequences of the primers used for reverse transcription quantitative PCR.

\begin{tabular}{|c|c|}
\hline Target & Sequences $\left(5^{\prime}-3^{\prime}\right)$ \\
\hline \multicolumn{2}{|c|}{ hsa-KCNQ1OT1 } \\
\hline Forward & GAACTCTGTTTTGTTTTCTGCTGC \\
\hline Reverse & CATAAATCCTGAAACAGACCCACTT \\
\hline \multicolumn{2}{|l|}{ hsa-GAS5 } \\
\hline Forward & AAGCCATTGGCACACAGGCATTAG \\
\hline Reverse & AGAACCATTAAGCTGGTCCAGGCA \\
\hline \multicolumn{2}{|c|}{ hsa-MINCR } \\
\hline Forward & CAGAAGAGCTTCATCGGCCC \\
\hline Reverse & TCACAGACGCACTCTTCCCA \\
\hline \multicolumn{2}{|r|}{ 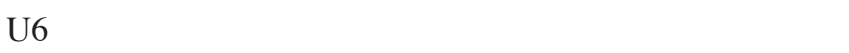 } \\
\hline Forward & CTCGCTTCGGCAGCACA \\
\hline Reverse & AACGCTTCACGAATTTGCGT \\
\hline \multicolumn{2}{|l|}{ GAPDH } \\
\hline Forward & GGAAGCTTGTCATCAATGGAAATC \\
\hline Reverse & TGATGACCCTTTTGGCTCCC \\
\hline \multicolumn{2}{|c|}{ hsa-miR-196b } \\
\hline Forward & CGGGCTAGGTAGTTTCCTGT \\
\hline Reverse & CAGCCACAAAAGAGCACAAT \\
\hline \multicolumn{2}{|c|}{ hsa-miR-642b } \\
\hline Forward & AGACACAUUUGGAGAGGGACCC \\
\hline Reverse & GAGUGUGCCCAAGAGAAAGUUU \\
\hline \multicolumn{2}{|c|}{ hsa-miR-3164 } \\
\hline Forward & TGTGACTTTAAGGGAAATGGCG \\
\hline Reverse & CTCTACAGCTATATTGCCAGCCAC \\
\hline \multicolumn{2}{|c|}{ hsa-miR-223-3p } \\
\hline Forward & TGTCAGTTTGTCAAATACC \\
\hline Reverse & AACTGGTGTCGTGAG \\
\hline \multicolumn{2}{|c|}{ hsa-miR-425 } \\
\hline Forward & ATGACACGATCACTCCCGTTG \\
\hline Reverse & GTGCAGGGTCCGAGGTATTC \\
\hline \multicolumn{2}{|c|}{ hsa-miR-122 } \\
\hline Forward & GCGTGATGGAGTGTGACAAT \\
\hline Reverse & GTGCAGGGTCCGAGGTATTC \\
\hline \multicolumn{2}{|c|}{ hsa-miR-483-3p } \\
\hline Forward & GGTGTCACTCCTCTCCTCC \\
\hline Reverse & CAGTGCGTGTCGTGGA \\
\hline \multicolumn{2}{|c|}{ hsa-miR-33b } \\
\hline Forward & GTGCATTGCTGTTGCATTGC \\
\hline Reverse & GTGCAGGGTCCGAGGT \\
\hline
\end{tabular}

miR, microRNA; GAS5, lncRNA growth arrest specific transcript 5; KCNQ1OT1, lncRNA KCNQ1 opposite strand/antisense transcript 1; MINCR, lncRNA MYC-induced long non-coding RNA.

(Hanbio Biotechnology Co., Ltd.). The miR-223-3p mimic and miR-223-3p mimic-negative control were constructed by GenePharma Co., Ltd. After 48 h of transfection, the Dual-Luciferase Reporter Assay system (cat. no. E1910; Promega Corporation) was used to measure the relative luciferase activity in each group and the data normalization was performed by comparing with the Renilla luciferase activity of KCNQ1OT1 WT + miR-223-3p mimic group. 
Table II. miRNAs with increased expression in patients with atrial fibrillation.

\begin{tabular}{|c|c|c|c|}
\hline Target & $\mid \mathrm{Log} 2 \mathrm{FCl}$ & $\mathrm{t}$ & P-value \\
\hline hsa-miR-33b & 0.869586221 & 2.365376187 & 0.02954409 \\
\hline hsa-miR-483-3p & 0.817867704 & 2.597063925 & 0.018293799 \\
\hline hsa-miR-122 & 0.772442509 & 2.541789208 & 0.020534659 \\
\hline hsa-miR-425 & 0.75856261 & 3.360950977 & 0.00351163 \\
\hline hsa-miR-196a & 0.708341176 & 2.607334223 & 0.017903851 \\
\hline hsa-miR-2276 & 0.660417901 & 2.909299897 & 0.009413676 \\
\hline hsa-miR-493 & 0.649498855 & 3.344417162 & 0.00364197 \\
\hline hsa-miR-4271 & 0.63060241 & 3.202575415 & 0.004974358 \\
\hline hsa-miR-1539 & 0.622510073 & 2.536416632 & 0.020765806 \\
\hline hsa-miR-4296 & 0.607087624 & 3.262255063 & 0.004363723 \\
\hline hsa-miR-587 & 0.606329463 & 4.071871233 & 0.000726406 \\
\hline hsa-miR-4298 & 0.602845228 & 2.412892937 & 0.026808013 \\
\hline hsa-miR-548p & 0.574869493 & 4.30723264 & 0.000431555 \\
\hline hsa-miR-605 & 0.550447818 & 2.738410535 & 0.013572845 \\
\hline hsa-let-7i & 0.549892958 & 3.102281544 & 0.006194148 \\
\hline hsa-miR-621 & 0.541751345 & 2.533866076 & 0.0208764 \\
\hline hsa-miR-3125 & 0.538749883 & 3.107985712 & 0.006117547 \\
\hline hsa-miR-3915 & 0.534540407 & 2.802455828 & 0.011840557 \\
\hline hsa-miR-3180 & 0.528461253 & 2.632995349 & 0.016963765 \\
\hline hsa-miR-492 & 0.52778329 & 3.813405954 & 0.001289044 \\
\hline hsa-miR-193b & 0.506441031 & 2.255811103 & 0.036874622 \\
\hline hsa-miR-641 & 0.505252296 & 2.308449626 & 0.033164494 \\
\hline hsa-miR-127-5p & 0.500711212 & 4.461170028 & 0.000307439 \\
\hline
\end{tabular}

miR, microRNA; FC, fold change.

Statistical analysis. Statistical analysis was performed using SPSS version 23 software (IBM Corp.). The categorical data are presented as the frequency and the continuous data are presented as the means \pm standard deviation. A $\chi^{2}$ test was used to determine the statistical differences between categorical data. For continuous and normally distributed data, statistical differences between two groups were compared using a unpaired two-tailed Student's t-test. $\mathrm{P}<0.05$ was considered to indicate a statistically significant difference.

\section{Results}

Microarray data. After screening, the miRNA microarray dataset GSE68475, which contained 10 patients with AF and 11 patients with SR, was selected for further analysis. The GSE68475 dataset, which was uploaded by Morishima et al (20), aimed to detect the miRNAs expression in human atrial appendages, and reported that miR-30d was essential for the electrical remodeling of AF. After preprocessing the miRNA microarray data according to the degree of samples dispersion, two samples from both the AF and SR groups were removed. Finally, eight samples from the AF group and nine samples from the SR group were obtained.

Identification of differentially expressed miRNAs in AF. miRNAs were screened for according to the threshold cutoff values used in the present study. The results identified 43 differentially expressed miRNAs in the AF and SR groups, including 23 upregulated miRNAs, such as miR-33b, miR-483-3p, miR-122 and miR-425 (Table II), and 20 downregulated miRNAs, such as miR-196b, miR-642b, miR-3164 and miR-223-3p (Table III). The heatmap and volcano map displaying the differentially expressed miRNAs were drawn using R software and are presented in Figs. 1 and 2.

Identification of differentially expressed miRNAs in RAA tissues from patients with $A F$. To verify the expression of the aforementioned differentially expressed miRNAs identified by screening the microarray data, RT-qPCR was used to determine the expression levels of the identified miRNAs in the RAA tissues from patients with AF or SR. In total, 16 patients (age range, 42-69 years) were used, including eight patients with persistent AF. The clinicopathological characteristics of the included patients are listed in Table IV, and no significant differences were observed in the sex, age, medical history or therapeutic regimen between the two groups.

Compared with patients with SR, the expression levels of miR-33b and miR-483-3p were found to be upregulated in the AF group, while the expression levels of miR-196b and miR-642b were downregulated. These findings were consistent with the microarray data. However, no significant differences were identified in the expression levels of miR-122, miR-425, 
Table III. miRNAs with decreased expression in patients with atrial fibrillation.

\begin{tabular}{|c|c|c|c|}
\hline Target & |Log2FCl & $\mathrm{t}$ & P-value \\
\hline hsa-miR-196b & -0.953061347 & -2.387936306 & 0.028214045 \\
\hline hsa-miR-642b & -0.684669912 & -5.232846332 & $5.78 \mathrm{E}-05$ \\
\hline hsa-miR-3164 & -0.667260096 & -3.14912112 & 0.005591877 \\
\hline hsa-miR-223-3p & -0.667052957 & -3.778345029 & 0.001393388 \\
\hline hsa-let-7e & -0.652894577 & -3.358265208 & 0.003532485 \\
\hline hsa-miR-4264 & -0.643875178 & -3.129959531 & 0.005831009 \\
\hline hsa-miR-301b & -0.613918625 & -3.965039772 & 0.000920629 \\
\hline hsa-miR-4254 & -0.601866722 & -3.137886674 & 0.005730892 \\
\hline hsa-miR-548o & -0.591841785 & -4.056385355 & 0.000751777 \\
\hline hsa-miR-556-3p & -0.585672054 & -2.622710761 & 0.017334758 \\
\hline hsa-miR-518c & -0.577279497 & -4.166962591 & 0.000588435 \\
\hline hsa-miR-628-5p & -0.575507912 & -5.543327212 & $3.00 \mathrm{E}-05$ \\
\hline hsa-miR-518b & -0.57442567 & -2.137014059 & 0.046696381 \\
\hline hsa-miR-130a & -0.570996268 & -2.417688503 & 0.026545462 \\
\hline hsa-miR-3678-3p & -0.566643877 & -2.618079507 & 0.017504327 \\
\hline hsa-miR-578 & -0.556014824 & -2.409518058 & 0.026994239 \\
\hline hsa-miR-889 & -0.544245666 & -6.228489463 & 7.35E-06 \\
\hline hsa-miR-433 & -0.524268417 & -2.193339731 & 0.041773836 \\
\hline hsa-miR-19a & -0.52202772 & -2.739368993 & 0.013545215 \\
\hline hsa-miR-553 & -0.520224796 & -5.015733997 & $9.19 \mathrm{E}-05$ \\
\hline
\end{tabular}

miR, microRNA; FC, fold change.

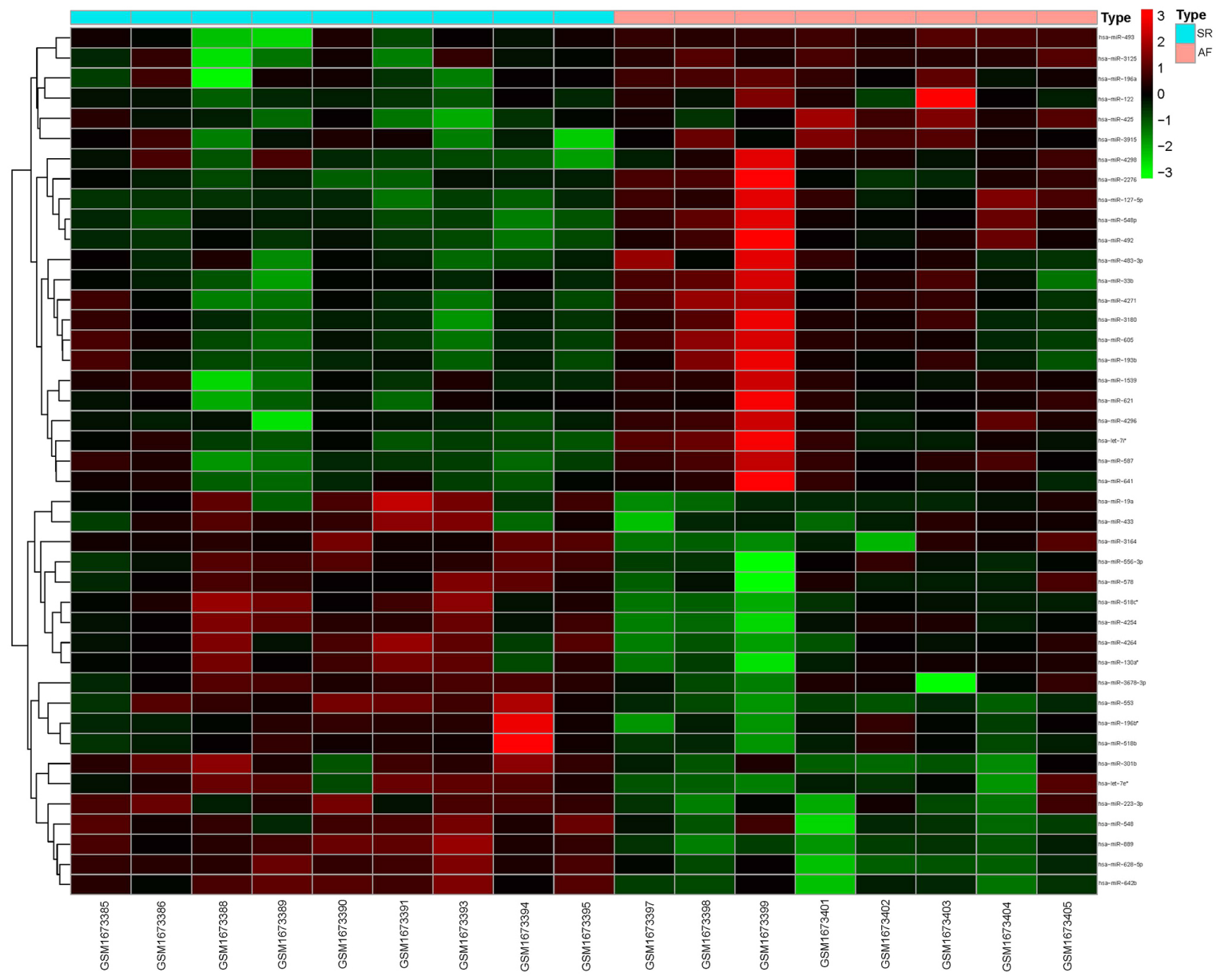

Figure 1. Heatmap of significantly differentially expressed microRNAs in AF. SR, sinus rhythm; AF, atrial fibrillation. 
Table IV. Clinicopathological characteristics of patients with atrial fibrillation.

\begin{tabular}{lccc}
\hline Characteristics & SR $(\mathrm{n}=8)$ & AF $(\mathrm{n}=8)$ & P-value \\
\hline Sex ratio, male/female & $5 / 3$ & $7 / 1$ & 0.119 \\
Age, years & $53.125 \pm 8.839$ & $54.125 \pm 7.338$ & 0.907 \\
Medical history & & & \\
Coronary heart disease (n) & 3 & 3 & 0.569 \\
Diabetes mellitus (n) & 0 & 4 & 0.200 \\
Hypertension (n) & 5 & & 0.334 \\
Therapy & & 1 & 0.569 \\
CABG & 3 & 2 & 0.467 \\
AVR & 0 & 3 & 0.619 \\
MVR & 5 & 2 & 0.467 \\
Combined valve replacement & 0 & & \\
\end{tabular}

SR, sinus rhythm as control; $\mathrm{AF}$, atrial fibrillation; $\mathrm{CABG}$, coronary artery bypass grafting; $\mathrm{AVR}$, aortic valve replacement; MVR, mitral valve replacement.

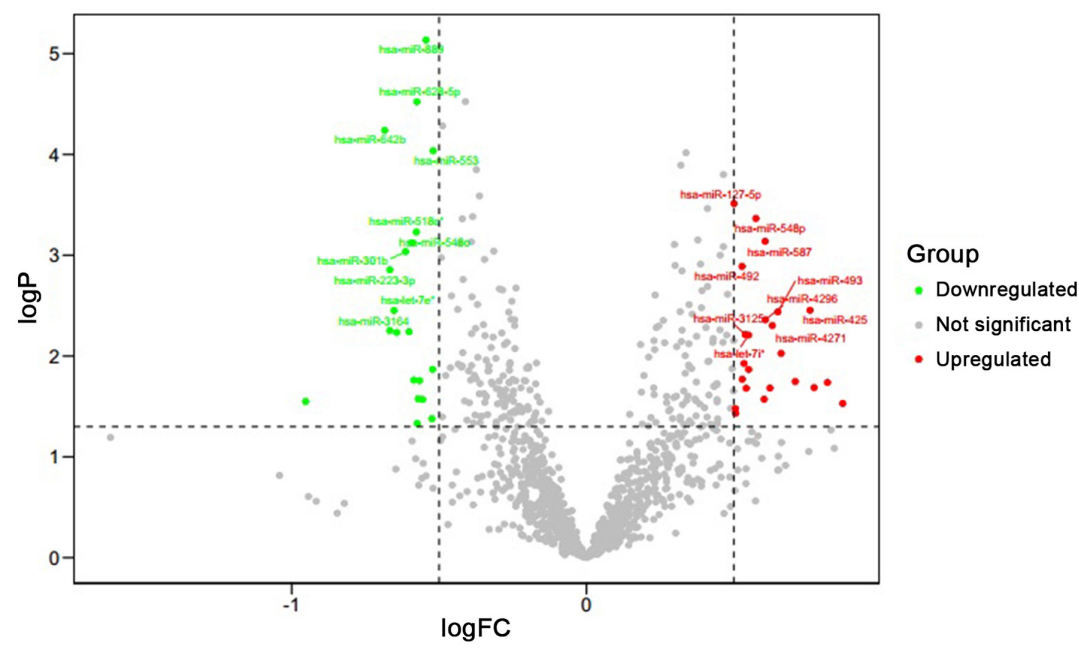

Figure 2. Volcano map of significantly differentially expressed miRNAs in AF. Green points show the downregulated miRNAs and red points show the upregulated miRNAs in AF samples. AF, atrial fibrillation; FC, fold change; miRNAs, microRNAs.

miR-3164 and miR-223-3p between the two groups (Fig. 3). Our previous study reported that miR-223-3p could mediate the generation of angiotensin (Ang)II-induced reactive oxygen species and thereby regulate the levels of oxidative stress (Weiran Dai; unpublished data). Oxidative stress is one of the important mechanisms required for the initiation and maintenance of AF. Zhang et al (21) reported that miR-223-3p regulates the activation of paired box 6 , resulting in apoptosis, a mechanism that plays a significant role in the progression of the prothrombotic state in AF. Novel evidence has suggested that the inhibition of miR-223-3p expression could upregulate the expression of FOXO3 and activate the autophagy pathway, thereby significantly inhibiting myocardial fibrosis and improving myocardial remodeling in AF (22). miR-223-3p may therefore have multiple regulatory mechanisms in AF and understanding the biological function and specific regulatory mechanisms of miR-223-3p in AF may be of great significance. For these reasons, miR-223-3p was selected for further analysis in the present study.
Functional enrichment analyses for target genes of $m i R-223-3 p$. According to the predictions of the bioinformatics database, 415 target genes were identified as having a potential regulatory relationship with miR-223-3p. To analyze the potential biological functions of target genes of miR-223-3p, GO functional terms and KEGG signaling pathways enriched by the target genes of miR-223-3p were identified using the Database for Annotation, Visualization and Integrated Discovery (version 6.8; https://david.ncifcrf.gov/). A total of $57 \mathrm{GO}$ functional terms and $21 \mathrm{KEGG}$ signaling pathways were obtained. For GO enrichment analysis, target genes of miR-223-3p were enriched in $20 \mathrm{BP}, 17 \mathrm{CC}$ and $20 \mathrm{MF}$, including 'nuclear function', 'transcription factor activity regulation', 'RNA polymerase II promoter', 'DNA template translation' and 'cytoplasmic composition' (Fig. 4). The results from the KEGG signaling pathway enrichment analysis revealed that target genes of miR-223-3p were enriched in 21 KEGG signaling pathways, including 'AMPK signaling 

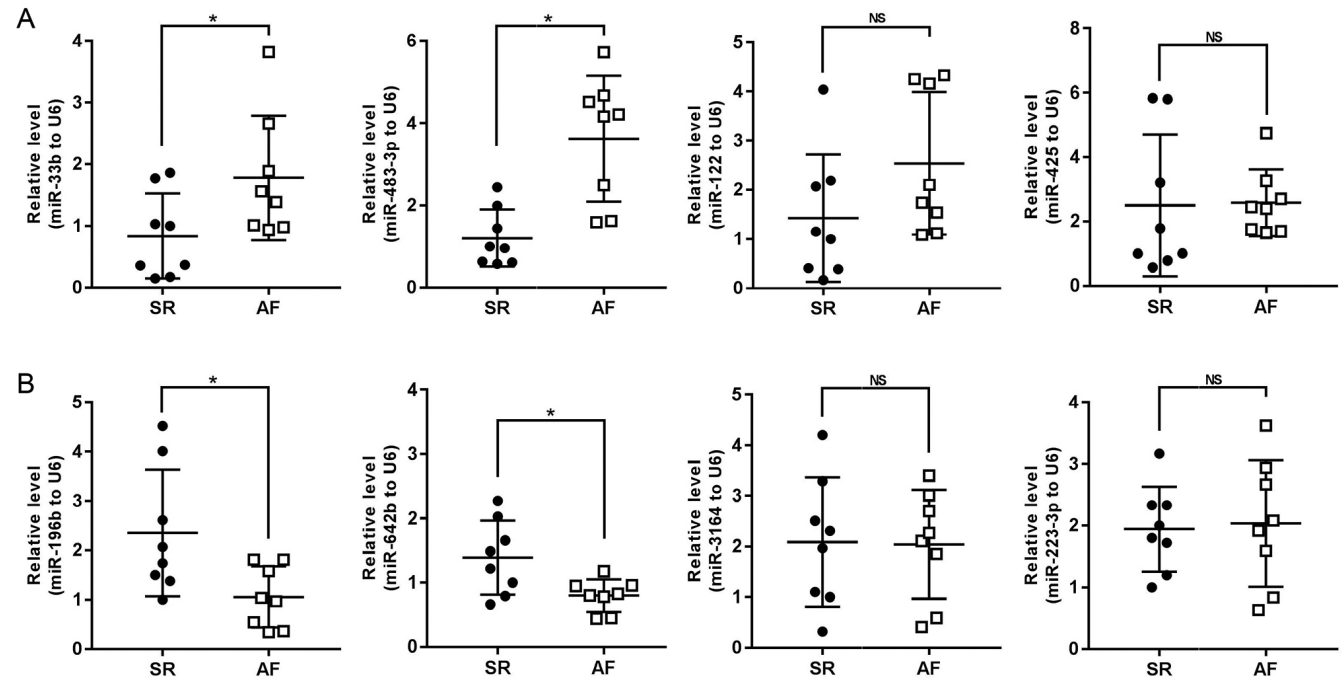

Figure 3. Expression of differentially expressed miRNAs selected from microarray were verified in right atrial appendage of patients with SR or chronic AF by RT-qPCR. (A) First four upregulated miRNAs were verified by RT-qPCR ( $\mathrm{n}=8$ per group). (B) First four downregulated miRNAs were verified by RT-qPCR (n=8 per group). "P<0.05 vs. SR group. SR, sinus rhythm; AF, atrial fibrillation; NS, non-significant; RT-qPCR, reverse transcription quantitative PCR; miRNAs, microRNAs.

pathway', 'stem cell pluripotency regulation', 'cGMP PKG signaling pathway' and 'insulin signaling pathway' (Fig. 5).

Identification of IncRNAs targeting miR-223-3p in the RAA tissues from patients with $A F$. According to the bioinformatics database prediction, 69 lncRNAs targeting miR-223-3p were identified, including lncRNA growth arrest specific transcript 5 (GAS5), lncRNA KCNQ1 opposite strand/antisense transcript 1 (KCNQ1OT1) and lncRNA MYC-induced long non-coding RNA (MINCR; Fig. 6A). As presented in Fig. 6B, the results from RT-qPCR analysis revealed that, compared with SR group, the expression level of KCNQ1OT1 was upregulated in RAA tissues from patients with AF. Conversely, the expression level of GAS5 was lower in patients with AF compared with that in patients with SR (Fig. 6C). However, no significant differences in the expression level of MINCR were identified between the two groups ( $P>0.05$; Fig. 6D). Furthermore, KCNQ1OT1 was identified as an AF-related IncRNA using GeneCards (https://www.genecards.org). KCNQ1OT1 was therefore selected for further analysis in the present study.

miR-223-3p is a direct target of KCNQ1OT1. To further investigate whether KCNQ1OT1 may directly mediate miR-223-3p, a dual luciferase reporter assay was performed. The results from the bioinformatics analysis suggested that miR-223-3p may be considered as a potential target of KCNQ1OT1, with two conserved putative binding sites identified between miR-223-3p and KCNQ1OT1 (Fig. 6E). The results from the dual luciferase reporter assay demonstrated that, compared with the KCNQ1OT1 WT + miR-223-3p mimic-NC group, the relative luciferase activity of the KCNQ1OT1 WT + miR-223-3p mimic group was significantly decreased $(1.0102 \pm 0.0234$ vs. $0.4903 \pm 0.0316$ ), while the decrease in relative luciferase activity was reversed in the KCNQ1OT1 MUT + miR-223-3p mimic and KCNQ1OT1 MUT + miR-223-3p mimic-NC groups (Fig. 6F). Taken together, these results suggested that
miR-223-3p may be a direct target of KCNQ1OT1, and that a ceRNA regulatory relationship may exist between KCNQ1OT1 and miR-223-3p in AF. These findings provided a novel potential target for the treatment of $\mathrm{AF}$ and might initiate further investigations.

\section{Discussion}

Despite the significant progress that has been made in the treatment of AF, AF continues to negatively impact individuals' lives, and the incidence increases with age. A recent study suggested that IncRNAs function as ceRNAs and could regulate miRNA expression and thereby affect the expression of target genes of miRNAs to contribute to the pathogenesis of AF (23). Therefore, screening for differentially expressed miRNAs using bioinformatics analysis remains an effective method to identify potential targets for future research into AF intervention. To the best of our knowledge, the present study was the first to identify and verify the specific regulatory relationship between KCNQ1OT1 and miR-223-3p using microarray data and may provide a promising target for future $\mathrm{AF}$ intervention experiments.

In cardiology, miR-223-3p has been shown to exert multiple regulatory effects. Previous studies have reported that miR-223-3p can regulate cell proliferation, apoptosis and necrosis at the post-transcriptional level via targeted inhibition mechanisms, and can also exert anti-inflammatory effects (24-26). A previous study reported that miR-223-3p could directly inhibit IкB kinase $\alpha$ to regulate the inflammatory response and necrosis induced by myocardial ischemia/reperfusion injury (27). Furthermore, another study suggested that circulating miR-223-3p levels might be used as a circulating marker of unstable plaques in coronary atherosclerotic heart disease (28). To fully understand the biological function of miR-223-3p, the present study performed GO functional term and KEGG signaling pathway enrichment analyses for potential miR-223-3p 


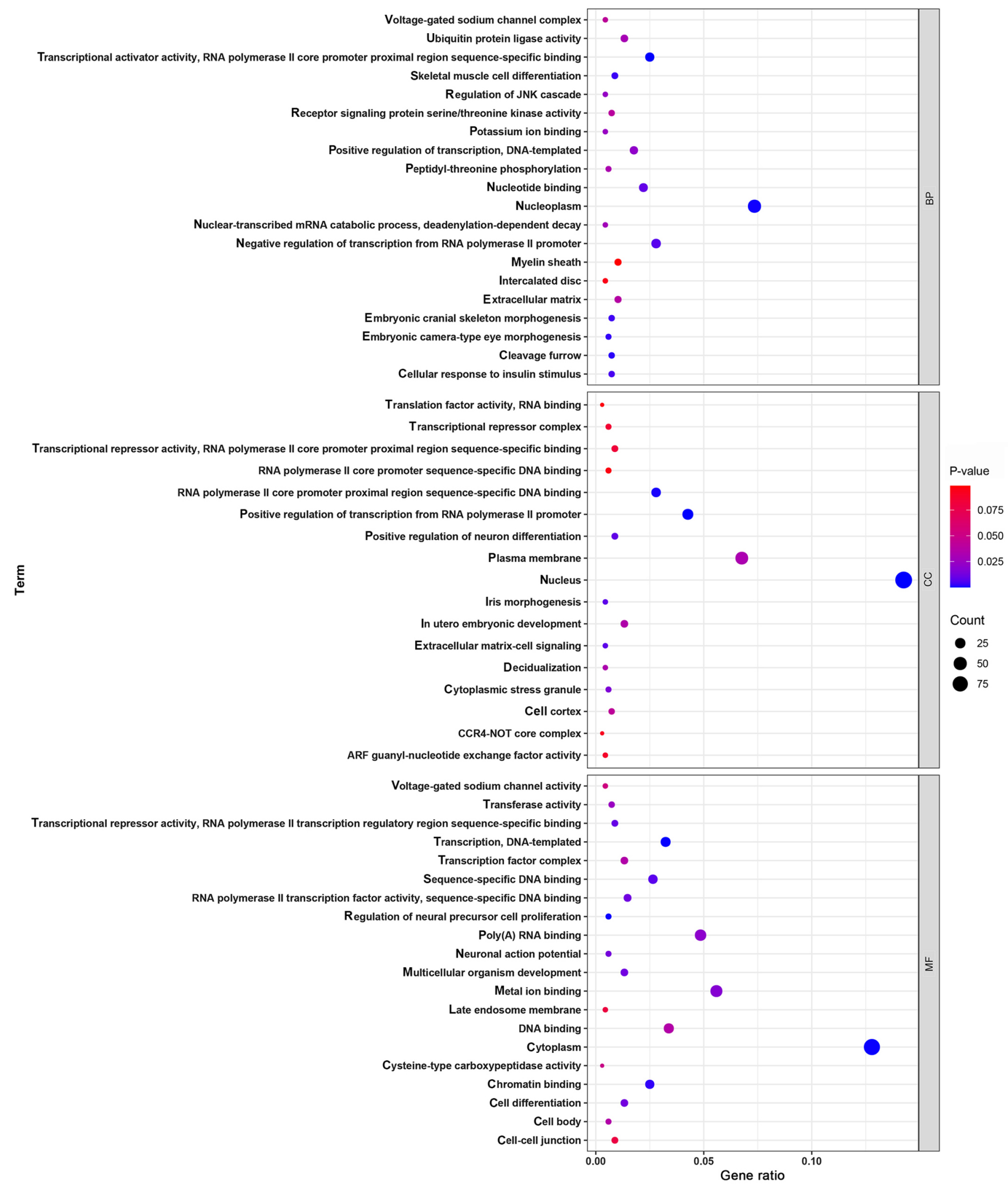

Figure 4. Gene Ontology function enrichment analysis of microRNA-223-3p target genes. BP, biological process; CC, cellular component; MF, molecular function.

target genes. The results from the GO functional term enrichment analysis revealed that target genes were mainly enriched in 'nuclear function', 'transcription factor activity regulation', 'RNA polymerase II promoter', 'DNA template translation' and 'cytoplasmic composition'. The results from the KEGG signaling pathway enrichment analysis demonstrated that target genes were mainly enriched in the 'AMPK signaling pathway', 'stem cell pluripotency regulation', 'cGMP PKG signaling pathway' and 'insulin signaling pathway'. 


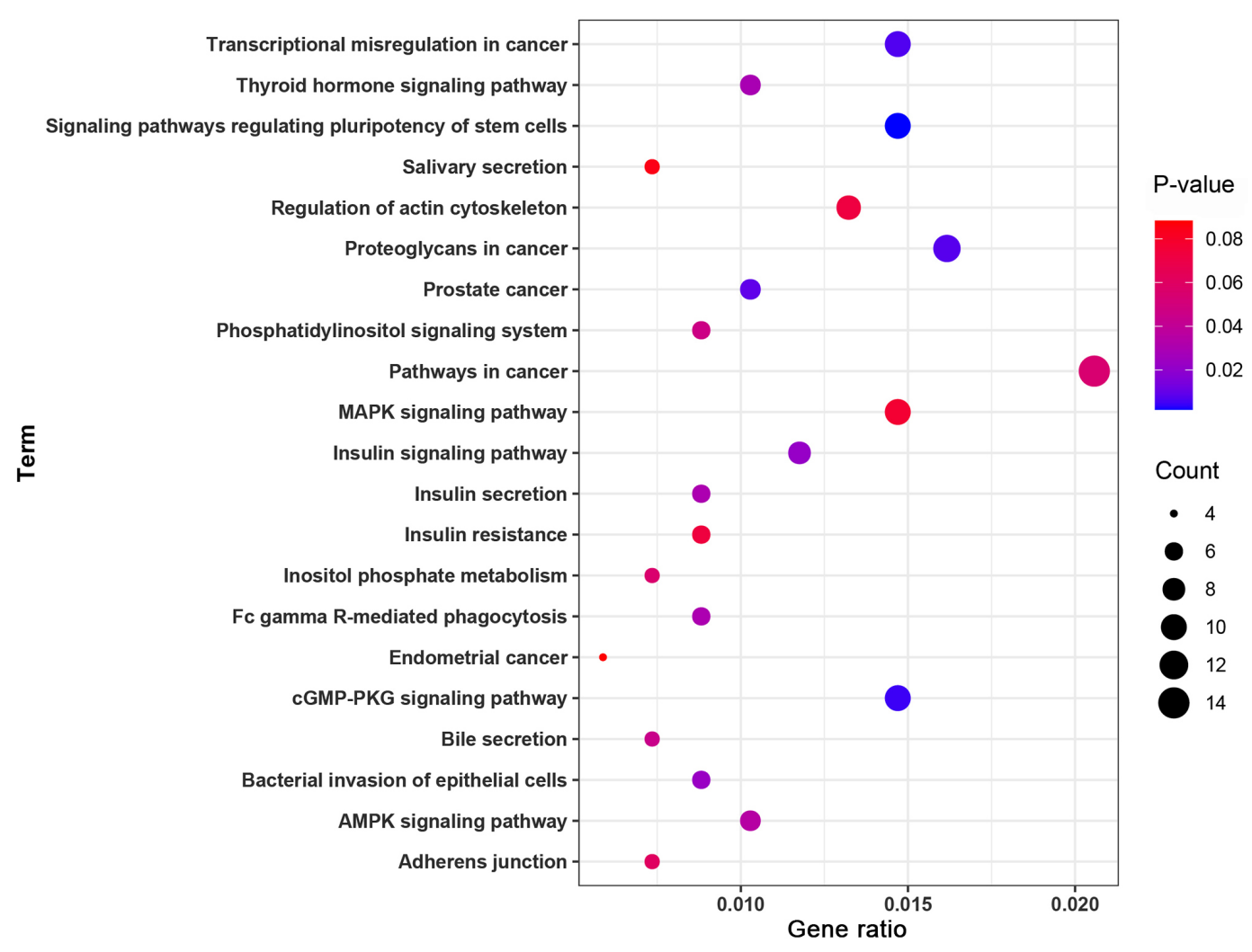

Figure 5. Kyoto Encyclopedia of Genes and Genomes pathway enrichment analysis of the target genes of microRNA-223-3p.

The AMPK signaling pathway is an important energy metabolism pathway, which plays an essential role in the regulation of cardiomyocyte energy homeostasis. AMPK regulates the metabolism of fatty acids and glycogen to maintain energy balance within the cell. Previous evidence also revealed that AMPK could regulate protein synthesis and cell proliferation via the eukaryotic translation elongation factor 2 and TSC complex subunit 2/mTOR signaling pathways and regulate blood flow via endothelial or neuronal nitric oxide synthase (29). In addition, the AMPK signaling pathway is also considered as a cardiac metabolite regulator. AMPK is a sensitive sensor of cell stress, which can be activated by phosphorylation of the upstream signal at position 172 threonine, and then exerts a protective effect by preventing metabolic stress, cell proliferation and hypertrophic remodeling, and regulating membrane electrophysiology. In addition, a previous study demonstrated that AMPK can also regulates the expression levels of $\mathrm{Ca}^{2+}$ channels and ion transporters to mediate intracellular $\mathrm{Ca}^{2+}$ signaling processing and transmission (30). In addition, abnormal $\mathrm{Ca}^{2+}$ handling may affect the action potential duration, which is an important mechanism of AF induction. Harada et al (31) report that downregulation of AMPK inhibits the abnormal $\mathrm{Ca}^{2+}$ transmission mediated by the integrity of atrial L-type $\mathrm{Ca}^{2+}$ channels, suggesting that the AMPK signaling pathway might play an important role in maintaining cardiac ion homeostasis and arrhythmias, including AF. Amongst the susceptibility models of AF, collagen-induced arthritis (CIA) is associated with the increased expression of inflammatory cytokines (interleukin-6 and tumor necrosis factor- $\alpha$ ), and it has been shown to lead to atrial remodeling and AF. Zhang et al (32) used a CIA rat model and demonstrated that CIA could induce atrial energy metabolism disorder by inhibiting the AMPK/PPARG coactivator $1 \alpha$ signaling pathway, while resveratrol could effectively reverse this inhibitory effect and reduce the susceptibility of AF in CIA model rats, suggesting that the increased risk of AF may be associated with the downregulation of the AMPK signaling pathway $(33,34)$. Another study demonstrated that fisetin can promote AMPK phosphorylation in the atrium, reducing AF susceptibility following myocardial infarction (35). The AMPK signaling pathway may therefore represent a potential research direction for future studies into AF intervention.

lncRNAs are important upstream regulators of the miRNA-associated ceRNA network by sponging miRNAs with related binding sites. In the present study, bioinformatics analysis predicted that $69 \mathrm{lncRNAs}$ could target miR-223-3p, including GAS5, KCNQ1OT1 and MINCR. The expression of these IncRNAs was subsequently verified in RAA tissues from patients with $\mathrm{AF}$, and the results demonstrated that compared with patients with SR, KCNQ1OT1 was upregulated and GAS5 was downregulated.

KCNQ1OT1 is located in the 15.5 region of the KCNQ1 locus on chromosome 11 (36). KCNQ1OT1 has been reported to be associated with numerous types of disease, including cardiovascular diseases (37). Li et al (38) demonstrated that KCNQ1OT1 could prevent myocardial ischemia/reperfusion injury following acute myocardial infarction by regulating adiponectin receptor 1 . Furthermore, KCNQ1OT1 was identified as an AF-related lncRNA using the GeneCard database. Consistent with the results from the present study, Shen et al (39) reported that KCNQ1OT1 expression is significantly upregulated in a time-dependent 


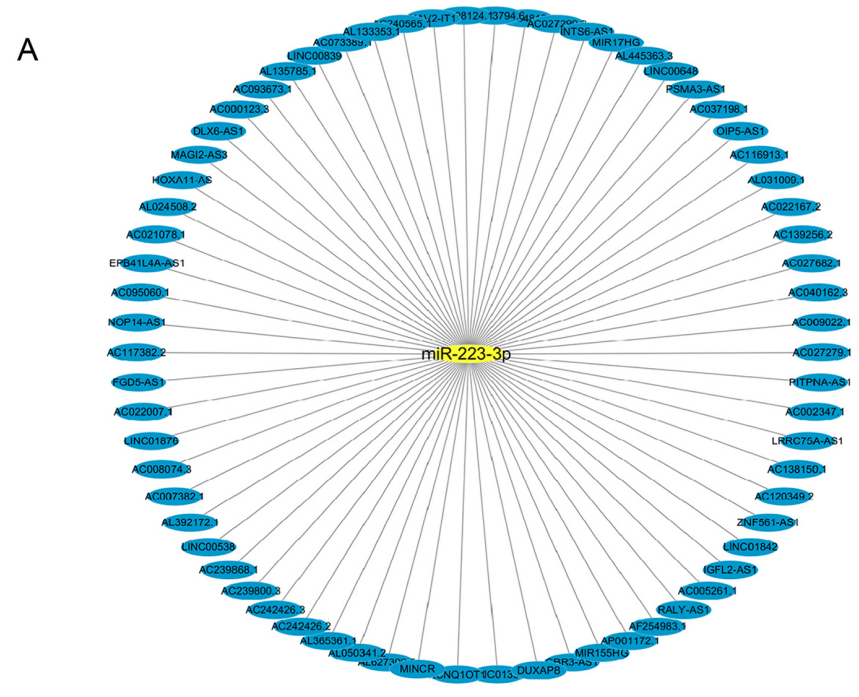

B

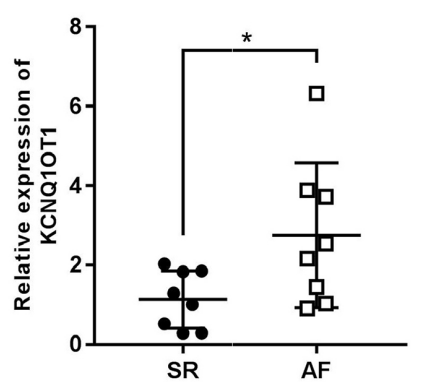

$E$
C

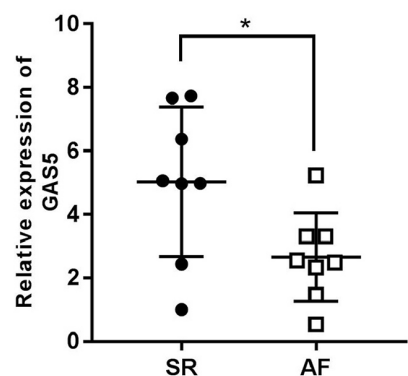

D

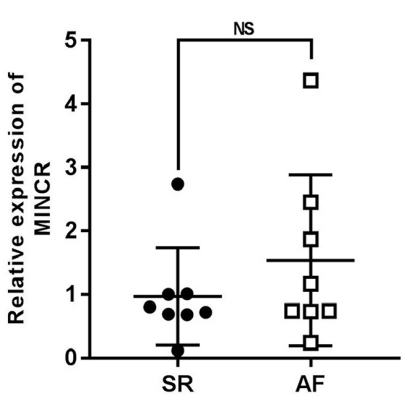

hsa-miR-223-3p

h-Inc-KCNQ1OT1
3'...ACCCCAUAAACUGUUUGACUGU...5'

I I | I I ।

5'...CGCUAUCUUUCUAUUAACUGACC...3'

$\begin{array}{cc}\text { hsa-miR-223-3p } & 3^{\prime} . . . \text { ACCCCAUAAACUGUUUGACUGU...5' } \\ \text { h-Inc-KCNQ10T1 } & \text { I I I I I I I I I I I I } \\ & \end{array}$

$\mathrm{F}$

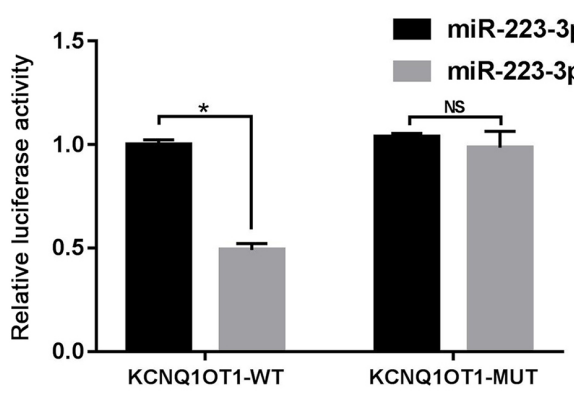

Figure 6. miR-223-3p is one direct target of KCNQ1OT1. (A) Bioinformatics database predicted the lncRNAs targeting miR-223-3p. (B-D) Expression of KCNQ1OT1, GAS5 and MINCR in RAA of patients with SR or AF ( $n=8$ per group); " $\mathrm{P}<0.05$ vs. SR group. (E) Binding site of KCNQ1OT1 and miR-223-3p. (F) Dual luciferase assay confirmed that miR-223-3p was one direct target of KCNQ1OT1 ( $n=3$ per group). "P<0.05 vs. KCNQ1OT1 WT + miR-223-3p NC group. SR, sinus rhythm; AF, atrial fibrillation; RAA, right atrial appendage; NS, non-significant; WT, wild type; MUT, mutant; miR, microRNA; NC, negative control; KCNQ1OT1, KCNQ1 opposite strand/antisense transcript 1.

manner in an angiotensin II (AngII) infusion mouse model. AngII treatment has been demonstrated to effectively increase AF susceptibility. AngII is therefore widely used in scientific research to establish an AF susceptibility model. In addition,
KCNQ1OT1 was shown to be an essential lncRNA involved in the regulation of a cardiovascular disease ceRNA network, and it could function as a molecular sponge by binding to miRNAs to regulate the expression of their downstream target genes. 
Yang et al (40) report that KCNQ1OT1 is highly expressed in patients with diabetic cardiomyopathy and that silencing KCNQ1OT1 successfully inhibits cell apoptosis by increasing the expression of miR-214-3p and then downregulating its target gene by acting as a ceRNA in vivo. Furthermore, a previous study indicated that KCNQ1OT1 might be involved in the occurrence of AF as a ceRNA by mediating the miR-384b/Ca ${ }^{2+}$ voltage-gated channel subunit $\alpha 1 \mathrm{C}$ axis (39). In the present study, the results from the dual luciferase reporter assay revealed that miR-223-3p could directly bind to KCNQ1OT1, suggesting that KCNQ1OT1 may regulate the expression of downstream genes targeted by miR-223-3p to exert its biological molecular effects. The findings from the present study may therefore provide a new promising target for AF intervention.

The lncRNA GAS5 plays an important role in a variety of cardiovascular diseases. For example, in hypertension, GAS5 has been considered as a new vascular remodeling regulator (41). In AF, GAS5 can reverse the proliferation of cardiac fibroblasts by inhibiting transforming growth factor $\beta$ receptor 1 , thus decreasing the synthesis of collagen fibers in the extracellular matrix and inhibiting the structural remodeling of the myocardium and the progression of AF (42). In addition, GAS5 has also been reported to serve as a ceRNA in cardiovascular diseases and inhibit the negative regulation of miRNAs on downstream gene expression. Zhou et al (43) demonstrated that GAS5, by acting as a ceRNA, can inhibit miR-21 via its molecular sponge action, thus regulating the expression of programmed cell death 4 , a direct target of miR-21, and mediating cell apoptosis in myocardial infarction. Increasing evidence has suggested that GAS5 might also act as a ceRNA and inhibit miR-223-3p, thereby regulating the expression of its downstream target genes (44). Similarly, Yao et al (45) reported that miR-223-3p could directly bind to the 3'-UTR of nicotinamide phosphoribosyltransferase (NAMPT) and that GAS5 could sponge miR-223-3p and relieve the inhibitory effect of miR-223-3p on NAMPT via regulating PI3K/AKT signaling. As there have been numerous studies demonstrating that GAS5 can directly target miR-223-3p $(44,45)$, the present study did not perform dual luciferase reporter assays to verify whether miR-223-3p was a target of GAS5. However, the underlying mechanism of GAS5 in AF based on the ceRNA theory has not been studied in detail, to the best of our knowledge, and will require further experimental verification.

The results from the present study provided novel promising targets for further experimentation to develop strategies for AF intervention. However, there were some limitations to the present study. Firstly, human samples were used to verify the differentially expressed miRNAs of interest found within the microarray dataset, in addition to the trends in the expression levels of upstream lncRNAs in AF. Unfortunately, the study did not perform in vitro transfection experiments to further verify the direct targeted regulatory relationship between the identified miRNAs and lncRNAs. Hence, the lack of functional in vitro experiments was a limitation to this study. The author's research group is currently performing in vivo and in vitro experiments to validate the results from the present study. Secondly, the sample size used in this study was relatively small, which may produce selection offset. Some evidence has suggested that miR-223-3p expression in
AF is controversial. For example, Wang et al (46) studied the miRNA transcriptome in AF using a tissue microarray and demonstrated that miR-223-3p expression level is upregulated in AF tissues. Conversely, a recent study using exosomes isolated from patients with $\mathrm{SR}$ and AF reported that compared patient with SR, miR-223-3p expression levels were downregulated in circulation expsomes of patients with AF (47). Thus, when the sample size used is relatively small and the reported expression of the gene is inconsistent with the literature, the selection offset should be expanded. Subsequently, further verification using larger sample sizes and multi-center research investigations is required.

In summary, the present study used microarray data on AF to identify significantly differently expressed miRNAs, and the results were subsequently verified in RAA tissues from patients with AF. Amongst the 43 significantly differently expressed miRNAs identified, miR-223-3p was discovered to be significantly upregulated in AF. Through GO functional term and KEGG signaling pathway enrichment analyses of the target genes of miR-223-3p, the 'AMPK signaling pathway' was identified as an important pathway. In addition, IncRNAs, including KCNQ1OT1, GAS5 and MINCR, were identified as targeting miR-223-3p. Furthermore, the results from dual luciferase reporter assay confirmed the existence of a targeted relationship between KCNQ1OT1 and miR-223-3p. These findings suggested that KCNQ1OT1 and GAS5 may serve crucial roles as ceRNAs in AF. These findings provided a solid theoretical basis for future intervention experiments and offered some potential novel targets for AF intervention. However, further transfection experiments both in vivo and in vitro should be performed to verify the findings from this study.

\section{Acknowledgements}

Not applicable.

\section{Funding}

This study was supported by the Natural Science Foundation of China (grant nos. 82060068 and 81760060).

\section{Availability of data and materials}

The GSE68475 dataset is available from the Gene Expression Omnibus website (http://www.ncbi.nlm.nih.gov/geo/).

\section{Authors' contributions}

WD and XC performed the experiments. WD wrote the manuscript. GZ and ZJ designed the experiments. ZJ carefully revised the important intellectual content of the manuscript. WD and GZ confirm the authenticity of all the raw data. All authors read and approved the final manuscript.

\section{Ethics approval and consent to participate}

The experimental protocol was approved by the Ethics Committee of The First Affiliated Hospital of Guangxi Medical University (Nanning, China). Each patient provided written informed consent. 


\section{Patient consent for publication}

Not applicable.

\section{Competing interests}

The authors declare that they have no competing interests.

\section{References}

1. Chugh SS, Havmoeller R, Narayanan K, Singh D, Rienstra M, Benjamin EJ, Gillum RF, Kim YH, McAnulty JH Jr, Zheng ZJ, et al: Worldwide epidemiology of atrial fibrillation: A Global Burden of Disease 2010 Study. Circulation 129: 837-847, 2014

2. Zimetbaum P: Atrial Fibrillation. Ann Intern Med 166 : ITC33-ITC48, 2017

3. Nattel S and Harada M: Atrial remodeling and atrial fibrillation: Recent advances and translational perspectives. J Am Coll Cardiol 63: 2335-2345, 2014.

4. Ferrari R, Bertini M, Blomstrom-Lundqvist C, Dobrev D Kirchhof P, Pappone C, Ravens U, Tamargo J, Tavazzi L and Vicedomini GG: An update on atrial fibrillation in 2014: From pathophysiology to treatment. Int J Cardiol 203: 22-29, 2016.

5. Liu Y, Shi Q, Ma Y and Liu Q: The role of immune cells in atria fibrillation. J Mol Cell Cardiol 123: 198-208, 2018.

6. Vyas V, Hunter RJ, Longhi MP and Finlay MC: Inflammation and adiposity: New frontiers in atrial fibrillation. Europace 22 1609-1618, 2020.

7. Arroyo AB, de Los Reyes-García AM, Rivera-Caravaca JM, Valledor P, García-Barberá N, Roldán V, Vicente V, Martínez C and González-Conejero R: miR-146a regulates neutrophil extracellular trap formation that predicts adverse cardiovascular events in patients with atrial fibrillation. Arterioscler Thromb Vasc Biol 38: 892-902, 2018.

8. Farazi TA, Hoell JI, Morozov P and Tuschl T: MicroRNAs in human cancer. Adv Exp Med Biol 774: 1-20, 2013.

9. Zhao Y, Yuan Y and Qiu C: Underexpression of CACNA1C caused by overexpression of microRNA-29a underlies the pathogenesis of atrial fibrillation. Med Sci Monit 22: 2175-2181 2016.

10. Cañón S, Caballero R, Herraiz-Martínez A, Pérez-Hernández M, López B, Atienza F, Jalife J, Hove-Madsen L, Delpón E and Bernad A: miR-208b upregulation interferes with calcium handling in HL-1 atrial myocytes: Implications in human chronic atrial fibrillation. J Mol Cell Cardiol 99: 162-173, 2016.

11. Lv X, Li J, Hu Y, Wang S, Yang C, Li C and Zhong G: Overexpression of miR-27b-3p targeting Wnt3a regulates the signaling pathway of $\mathrm{Wnt} / \beta$-catenin and attenuates atrial fibrosis in rats with atrial fibrillation. Oxid Med Cell Longev 2019: 5703764, 2019.

12. Kong F, Jin J, Lv X, Han Y, Liang X, Gao Y and Duan X: Long noncoding RNA RMRP upregulation aggravates myocardial ischemia-reperfusion injury by sponging miR-206 to target ATG3 expression. Biomed Pharmacother 109: 716-725, 2019.

13. Salmena L, Poliseno L, Tay Y, Kats L and Pandolfi PP: A ceRNA hypothesis: the Rosetta Stone of a hidden RNA language? Cell 146: 353-358, 2011.

14. Lou W, Ding B and Fu P: Pseudogene-derived lncRNAs and their miRNA sponging mechanism in human cancer. Front Cell Dev Biol 8: 85, 2020.

15. Wang LY, Shen H, Yang Q, Min J, Wang Q, Xi W, Yin L, Le SG, Zhang YF, Xiao J, et al: LncRNA-LINC00472 contributes to the pathogenesis of atrial fibrillation (Af) by reducing expression of JP2 and RyR2 via miR-24. Biomed Pharmacother 120: 109364 2019.

16. Kirchhof P, Benussi S, Kotecha D, Ahlsson A, Atar D, Casadei B, Castella M, Diener HC, Heidbuchel H, Hendriks J, et al; ESC Scientific Document Group: 2016 ESC Guidelines for the management of atrial fibrillation developed in collaboration with EACTS. Eur Heart J 37: 2893-2962, 2016

17. Livak KJ and Schmittgen TD: Analysis of relative gene expression data using real-time quantitative PCR and the 2(-Delta Delta C(T)) method. Methods 25: 402-408, 2001.

18. Gene Ontology Consortium: The Gene Ontology (GO) project in 2006. Nucleic Acids Res 34: D322-D326, 2006.
19. Kanehisa M, Sato Y, Kawashima M,Furumichi M and Tanabe M: KEGG as a reference resource for gene and protein annotation. Nucleic Acids Res 44 (D1): D457-D462, 2016.

20. Morishima M, Iwata E, Nakada C, Tsukamoto Y, Takanari H, Miyamoto S, Moriyama M and Ono K: Atrial Fibrillation-Mediated Upregulation of miR-30d Regulates Myocardial Electrical Remodeling of the G-Protein-Gated $\mathrm{K}(+)$ Channel, IK.ACh. Circ J 80: 1346-1355, 2016.

21. Zhang H, Yang G, Zhong N, Shan J, Li X, Wu Y, Xu Y and Yuan Y: Possible key microRNAs and corresponding molecular mechanisms for atrial fibrillation. Anatol J Cardiol 23: 324-333, 2020.

22. Hu J, Wang X, Cui X, Kuang W, Li D and Wang J: Quercetin prevents isoprenaline-induced myocardial fibrosis by promoting autophagy via regulating miR-223-3p/FOXO3. Cell Cycle 20: $1253-1269,2021$.

23. Li Z, Wang X, Wang W, Du J, Wei J, Zhang Y, Wang J and Hou Y: Altered long non-coding RNA expression profile in rabbit atria with atrial fibrillation: TCONS_00075467 modulates atrial electrical remodeling by sponging miR-328 to regulate CACNA1C. J Mol Cell Cardiol 108: 73-85, 2017.

24. Ji Q, Xu X, Song Q, Xu Y, Tai Y, Goodman SB, Bi W, Xu M, Jiao S, Maloney WJ, et al: miR-223-3p inhibits human osteosarcoma metastasis and progression by directly targeting CDH6. Mol Ther 26: 1299-1312, 2018.

25. Matsuzaki J and Ochiya T: Extracellular microRNAs and oxidative stress in liver injury: A systematic mini review. J Clin Biochem Nutr 63: 6-11, 2018.

26. Boxberger N, Hecker $M$ and Zettl UK: Dysregulation of Inflammasome Priming and Activation by MicroRNAs in Human Immune-Mediated Diseases. J Immunol 202: 2177-2187, 2019.

27. Qin D, Wang X, Li Y, Yang L, Wang R, Peng J, Essandoh K, Mu X, Peng T, Han Q, et al: MicroRNA-223-5p and -3p Cooperatively Suppress Necroptosis in Ischemic/Reperfused Hearts. J Biol Chem 291: 20247-20259, 2016.

28. Singh S, de Ronde MWJ, Kok MGM, Beijk MA, De Winter RJ, van der Wal AC, Sondermeijer BM, Meijers JCM, Creemers EE and Pinto-Sietsma SJ: MiR-223-3p and miR-122-5p as circulating biomarkers for plaque instability. Open Heart 7: e001223, 2020.

29. Hardie DG: The AMP-activated protein kinase pathway--new players upstream and downstream. J Cell Sci 117: 5479-5487, 2004

30. Chakraborty P, Nattel S and Nanthakumar K: Linking cellular energy state to atrial fibrillation pathogenesis: Potential role of adenosine monophosphate-activated protein kinase. Heart Rhythm 17: 1398-1404, 2020.

31. Harada M, Tadevosyan A, Qi X, Xiao J, Liu T, Voigt N, Karck M, Kamler M, Kodama I, Murohara T, et al: Atrial Fibrillation Activates AMP-Dependent Protein Kinase and its Regulation of Cellular Calcium Handling: Potential Role in Metabolic Adaptation and Prevention of Progression. J Am Coll Cardiol 66: 47-58, 2015

32. Zhang Y, Zhang S, Liu Z, Zhao X, Yuan Y, Sheng L and Li Y: Correction to: Resveratrol prevents atrial fibrillation by inhibiting atrial structural and metabolic remodeling in collagen-induced arthritis rats. Naunyn Schmiedebergs Arch Pharmacol 393 : 927-928, 2020. Erratum for: Naunyn Schmiedebergs Arch Pharmacol 391: 1179-1190, 2018

33. Abe I, Teshima Y, Kondo H, Kaku H, Kira S, Ikebe Y, Saito S, Fukui A, Shinohara T, Yufu K, et al: Association of fibrotic remodeling and cytokines/chemokines content in epicardial adipose tissue with atrial myocardial fibrosis in patients with atrial fibrillation. Heart Rhythm 15: 1717-1727, 2018.

34. Maesen B, Nijs J, Maessen J, Allessie M and Schotten U: Post-operative atrial fibrillation: A maze of mechanisms. Europace 14: 159-174, 2012

35. Liu L, Gan S, Li B, Ge X, Yu H and Zhou H: Fisetin Alleviates Atrial Inflammation, Remodeling, and Vulnerability to Atrial Fibrillation after Myocardial Infarction. Int Heart J 60: 1398-1406, 2019

36. Kanduri C: Kcnq1ot1: A chromatin regulatory RNA. Semin Cell Dev Biol 22: 343-350, 2011

37. Jiang Y, Du W, Chu Q, Qin Y, Tuguzbaeva G, Wang H, Li A, Li G, Li Y, Chai L, et al: Downregulation of Long Non-Coding RNA Kcnq1ot1: An Important Mechanism of Arsenic Trioxide-Induced Long QT Syndrome. Cell Physiol Biochem 45: 192-202, 2018.

38. Li X, Dai Y, Yan S, Shi Y, Han B, Li J, Cha L and Mu J: Down-regulation of IncRNA KCNQ1OT1 protects against myocardial ischemia/reperfusion injury following acute myocardial infarction. Biochem Biophys Res Commun 491: $1026-1033,2017$ 
39. Shen C, Kong B, Liu Y, Xiong L, Shuai W, Wang G, Quan D and Huang H: YY1-induced upregulation of lncRNA KCNQ1OT1 regulates angiotensin II-induced atrial fibrillation by modulating miR-384b/CACNA1C axis. Biochem Biophys Res Commun 505: 134-140, 2018

40. Yang F, Qin Y, Wang Y, Li A, Lv J, Sun X, Che H, Han T, Meng S, Bai Y, et al: LncRNA KCNQ1OT1 Mediates Pyroptosis in Diabetic Cardiomyopathy. Cell Physiol Biochem 50: 1230-1244, 2018.

41. Wang YN, Shan K, Yao MD, Yao J, Wang JJ, Li X, Liu B, Zhang YY, Ji Y, Jiang Q, et al: Long Noncoding RNA-GAS5: A Novel Regulator of Hypertension-Induced Vascular Remodeling. Hypertension 68: 736-748, 2016.

42. Lu J, Xu FQ, Guo JJ, Lin PL, Meng Z, Hu LG, Li J, Li D, Lu XH and An Y: Long noncoding RNA GAS5 attenuates cardiac fibroblast proliferation in atrial fibrillation via repressing ALK5. Eur Rev Med Pharmacol Sci 23: 7605-7610, 2019.

43. Zhou XH, Chai HX, Bai M and Zhang Z: LncRNA-GAS5 regulates PDCD4 expression and mediates myocardial infarction-induced cardiomyocytes apoptosis via targeting miR-21. Cell Cycle 19: 1363-1377, 2020.
44. Dong X, Kong C, Liu X, Bi J, Li Z, Li Z, Zhu Y and Zhang Z: GAS5 functions as a ceRNA to regulate hZIP1 expression by sponging miR-223 in clear cell renal cell carcinoma. Am J Cancer Res 8: 1414-1426, 2018.

45. Yao J, Shi Z, Ma X, Xu D and Ming G: lncRNA GAS5/miR-223/NAMPT axis modulates the cell proliferation and senescence of endothelial progenitor cells through PI3K/AKT signaling. J Cell Biochem 120: 14518-14530, 2019.

46. Wang JG, Meng X, Han J, Li Y, Luo TG, Wang J, Xin M and Xi JZ: Differential expressions of miRNAs in patients with nonvalvular atrial fibrillation. Zhonghua Yi Xue Za Zhi 92: 1816-1819, 2012 (In Chinese).

47. Wang S, Min J, Yu Y, Yin L, Wang Q, Shen H, Yang J, Zhang P, Xiao J and Wang Z: Differentially expressed miRNAs in circulating exosomes between atrial fibrillation and sinus rhythm. J Thorac Dis 11: 4337-4348, 2019.

c) (i) This work is licensed under a Creative Commons Attribution 4.0 International (CC BY 4.0) License. 